\title{
Alterações Fisiológicas Relacionadas à Síndrome da tensão Pré-Menstrual na Vida da Mulher
}

\author{
Natália Alves Mendes ${ }^{1}$; Anderson Pereira de Souza ${ }^{2}$
}

Resumo: A Síndrome Pré-Menstrual (SPM) é caracterizada por mais de cem sinais e sintomas físicos e psicológicos diferentes, o que torna difícil defini-la cientificamente. Objetivo: identificar as possíveis consequências da síndrome da tensão pré-menstrual na vida das mulheres, especificamente, em mulheres estudantes do nível superior. Metodologia: A presente pesquisa se classifica como revisão não sistemática da literatura. A amostra foi constituída por artigos, teses e dissertações indexados nas bases de dados MEDline, Scielo, National Center for Biotechnology Information e Asian Nursing Research. Inicialmente surgiram cerca de 7.200 resultados, contudo foram selecionados apenas 38 e após leitura dos resumos dos mesmos, foram excluídas 9 publicações. Resultados e Discussões: Após análise do conteúdo dos artigos foram criadas duas categorias de discussão: Sintomas da SPTM e consequências no cotidiano das universitárias e Alternativas para controle dos sintomas. Considerações Finais: A SPM pode acarretar graves prejuízos no contexto social (família/trabalho/estudo) e contexto pessoal.

Palavras-Chave: Síndrome Pré-menstrual, consequências, universidade.

\section{Physiological Changes Related to Premenstrual Tension Syndrome in Women's Life}

\begin{abstract}
Premenstrual syndrome is characterized by more than a hundred physical and psychological different symptoms, what Objective: To identify possible premenstrual syndrome consequences, specifically, in high level women students. Methodology: This research is classified as nonsystematic literature review. The sample was constituted by articles, theses and dissertations indexed in databases as MEDline, Scielo, National Center for Biotechnology Information and Asian Nursing Research. At first time emerged about 7.200 results, although it was selected only 38 and after abstract reading of the articles, it was excluded 9 publications. Results and discussions: After content analyses it was created two discussions categories: PTS symptoms and consequences in high level students daily, and Methods to symptoms control. Final Considerations: The PTS may entail severe damages in social context (family/work/study) and individual context.
\end{abstract}

Keywords: Premenstrual syndrome, consequences, university.

\section{Introdução}

O ciclo menstrual dura, em média, 28 dias e pode ser dividido em três fases diferentes: fase folicular, ovulação, e a fase lútea. A fase folicular começa no primeiro dia da menstruação e dura de 8 a 23 dias; ovulação pode durar até 3 dias; e a fase lútea dura desde o final da ovulação até o início do fluxo menstrual. ${ }^{1}$

\footnotetext{
${ }^{1}$ Acadêmica do curso de Enfermagem da Faculdade Independente do Nordeste. E-mail: natalia_enfa@ hotmail.com;

${ }^{2}$ Docente e mestre do colegiado do curso de Enfermagem da Faculdade Independente do Nordeste; andersonpereira@ fainor.com.br.
} 
Id on Line Revista Multidisciplinar e de Psicologia

Id on Line Multidisciplinary and Psycology Journal

O período pré-menstrual é uma fase de vulnerabilidade para a ocorrência de sintomas físicos e psíquicos que geralmente aparecem uma semana antes da menstruação e desaparecem com o início do fluxo menstrual. ${ }^{2}$

A Síndrome da Tensão Pré-Menstrual ou Síndrome Pré-Menstrual (SPM) é caracterizada por mais de cem sinais e sintomas físicos e psicológicos diferentes, o que torna difícil defini-la cientificamente. ${ }^{3}$ Nos Estados Unidos da América (EUA), a prevalência de atitudes que relacionam o ciclo menstrual a alterações adversas do humor e do comportamento é tão alta que desvia as percepções femininas, a forma que as mulheres relatam os sintomas à alguns pesquisadores e os fatores aos quais atribuem sentimentos negativos. Deste modo, a identificação precoce e o tratamento adequado da SPM pode reduzir a probabilidade de sintomas crônicos e recorrentes. ${ }^{4}$

Alguns fatores sociais e culturais interferem na forma, como uma mulher percebe a SPM, popularmente conhecida por (TPM) e seus efeitos. Embora as circunstâncias envolvidas e a intensidade do impacto sofrido possam diferir de uma mulher para a outra, o fato é de que a síndrome acomete mulheres de todo mundo. Entre $75 \%$ a $95 \%$ de mulheres em idade reprodutiva que tem o ciclo menstrual regular, irão apresentar algum tipo de sintoma prémenstrual de intensidade leve. ${ }^{5-6}$

Desta forma, com base na diversidade de sinais, a SPM pode ser caracterizada por um conjunto de sintomas físicos, psicológicos e comportamentais, que começam uma semana anterior à menstruação e aliviam apenas com o início do fluxo menstrual. ${ }^{1}$

Esse estudo assume grande relevância à medida que pretende identificar as possíveis consequências da síndrome da tensão pré-menstrual na vida das mulheres, especificamente, em mulheres estudantes do nível superior, quando esses sintomas interferem na vida social, acadêmica e pessoal. Além disso, espera-se com esse trabalho contribuir para o aprimoramento do conhecimento dos profissionais da área de saúde, em específico, os de enfermagem especializados na área de saúde da mulher, bem como para os familiares e, principalmente favorecer a melhoria para o bem-estar feminino. 
Id on Line Revista Multidisciplinar e de Psicologia

Id on Line Multidisciplinary and Psycology Journal

\section{Metodologia}

A presente pesquisa se classifica como revisão não sistemática da literatura, tem natureza descritiva e exploratória, uma vez que faz uso da descrição, coleta e registro de dados. O caráter exploratório da pesquisa visa proporcionar uma visão geral sobre determinado fato e permite ao pesquisador uma maior familiaridade com o fenômeno que está sendo investigado. ${ }^{7}$

A amostra foi constituída por artigos, teses e dissertações indexados nas bases de dados como: MEDline, Scielo, National Center for Biotechnology Information e Asian Nursing Research, empregando-se os descritores: "Síndrome da tensão pré-menstrual", "universitárias", "nível superior", "tensão pré-menstrual", "consequências", "cotidiano". Os artigos foram coletados nas bases de dados entre os meses de agosto e setembro, do ano de 2016.

Foi utilizado o indicador booleano "and", foi utilizada restrição de língua inglesa ou portuguesa, porém com restrição de ano (2010 à 2016). Inicialmente surgiram cerca de 7.200 resultados, sendo que foram selecionados apenas 38 e após leitura dos resumos dos mesmos, foram excluídas 9 publicações uma vez que eram estudos repetidos, ou que não tinham relação direta com a questão de pesquisa.

Após análise do conteúdo dos artigos, foram criados dois eixos temáticos, a saber: 1 . Sintomas da SPTM e consequências no cotidiano das universitárias; 2. Alternativas para controle dos sintomas. ${ }^{8}$

\section{Resultados}

As 29 publicações analisadas eram teses, dissertações ou artigos disponíveis na íntegra. As publicações internacionais totalizaram 11, estavam escritas em língua inglesa e foram pesquisas oriundas de países como: Alemanha, Canadá, USA, França, etc., especificamente sobre SPM em universitárias foram encontradas apenas três estudos realizados em Portugal, Egito e na Etiópia, em 2011, 2013 e 2014 respectivamente.

As publicações nacionais aproveitadas totalizaram 18 e foram desenvolvidas em diversos estados do Brasil, como: Minas Gerais (MG), São Paulo (SP), Rio Grande do Sul (RS), Pernambuco (PE), Ceará (CE), etc., no entanto especificamente sobre SPM em universitárias 
Id on Line Revista Multidisciplinar e de Psicologia

Id on Line Multidisciplinary and Psycology Journal

apenas 6 publicações foram encontradas, sendo realizadas nos seguintes estados: MG uma publicação em 2015, RS uma publicação em 2014, CE duas publicações em 2010 e 2014, PE uma publicação em 2010.

\title{
Discussões
}

\section{Sintomas da SPTM e consequências no cotidiano das universitárias}

A presença de sintomas pré-menstruais graves, em mulheres em idade reprodutiva/fértil são muito comuns, ocorrendo em cerca de 3 à $8 \%$ deste público e caracterizando a Síndrome Pré-Mentrual (SPM). ${ }^{9}$

\begin{abstract}
A SPM é uma síndrome que dependendo da intensidade e duração das suas manfestações pode exigir atenção especial e tratamento dirigido. É mais conhecida como tensão pré-menstrual (TPM), e caracteriza-se como um conjunto de sintomas físicos, emocionais e comportamentais, que ocorrem de forma cíclica no período fértil, e podem ser variáveis na quantidade e na intensidade. ${ }^{10: 1538}$
\end{abstract}

Sintomas estes que podem culminar em fortes impactos no cotidiano das mulheres. De acordo a intensidade dos sintomas apresentados, a SPM pode ser classificada como mais severa, sendo chamada de "premenstrual dysphoric disorder (PMDD)", que pode ser traduzido como Transtorno Disfórico Pré-menstrual (TDPM). ${ }^{9}$

Existem diferenças importantes entre a SPM e TDPM, diferenças que não estão presentes meramente na severidade dos sintomas, mas também na quantidade de sintomas apresentados. Para diagnóstico da TDPM são necessários pelo menos cinco sintomas. No entanto para a SPM, sugere-se como critério de diagnóstico a existência de apenas um dos sintomas da lista abaixo, além disso, este sintoma deve causar "prejuízo nas atividades rotineiras, no trabalho, na escola ou no convívio social." $9,6: 3$

1 - labilidade afetiva; 2 - irritabilidade ou aumento dos conflitos interpessoais; 3 humor deprimido; 4 - ansiedade ou tensão; 5 - diminuição do interesse; 6 - dificuldade de concentração; 7 - letargia, fadiga ou baixa disposição; 8 - mudanças no apetite; 9 - insônia ou disposição melhorada para o sono; 10 - sensação de autocontrole; 11 outros sintomas físicos (dor e mastalgia)". 9:2 
Os diversos estudos que avaliaram a presença da SPM na vida de mulheres em idade fértil mostraram que os principais sintomas presentes são os sintomas físicos como: mastalgia, cólicas, edema, cefaleia, e os sintomas emocionais como: irritabilidade, ansiedade, tensão, sintomas depressivos. ${ }^{1,6,10-13}$

Os estudos específicos que avaliaram a ocorrência dos sintomas da SPM em acadêmicas de diversos cursos como: enfermagem, fisioterapia, medicina, também relataram a presença dos sintomas supracitados, como é possível visualizar no quadro abaixo (quadro 01). ${ }^{6,10,12,13,14}$

Quadro 01: Sintomas da SPM mais frequentes em acadêmicas do ensino superior.

\begin{tabular}{|ll|}
\hline Sintomas Físicos & Sintomas Emocionais \\
\hline Mastalgia $(68,2 \%-77 \%)$ & Irritabilidade $(55 \%-86 \%)$ \\
Cólicas $(62,3 \%-74 \%)$ & Humor deprimido $(48,2 \%-74,6 \%)$ \\
Edema em alguma parte do corpo $(49,2 \%-$ & Dificuldade de concentração (47\% - \\
$81,5 \%)$ & $55,4 \%)$ \\
Cefaleia $(46,9 \%-62 \%)$ & Ansiedade $(55,4 \%-60,7 \%)$ \\
Cansaço $(17 \%-40,8 \%)$ & \\
\hline
\end{tabular}

Fonte: Elaboração própria

Destaca-se que os valores apresentados foram obtidos em estudos transversais, com amostras variáveis entre 200 e 600 mulheres. Na categoria sintomas físicos, a presença de edema em alguma parte do corpo, principalmente na região abdominal, foi mais citada pelas universitárias, seguida de mastalgia. Na categoria sintomas emocionais a irritabilidade ganhou destaque, seguida do humor deprimido.

Tanto a presença de edema quanto da mastalgia tratam-se de alterações fisiológicas que não trazem prejuízos diretos ao organismo e ocorrem "devido ao hormônio progesterona que gera danos nos vasos sanguíneos prejudicando a drenagem e a retenção hídrica, o que causa a sensação de ganho de peso" 10:1539

Vale pontuar que em relação em um estudo realizado com 226 estudantes a mastalgia além de ser tida como "desconforto físico, pode prejudicar no relacionamento amoroso, bem 
Id on Line Revista Multidisciplinar e de Psicologia

Id on Line Multidisciplinary and Psycology Journal

como trazer alguns temores quanto à presença de doenças mamárias, inclusive preocupação com o câncer das mamas."12:552

Nos aspectos emocionais, a irritabilidade está associada a menor resposta cerebral, ao neurotransmissor inibitório Acido Gama-aminobutírico (GABA) ${ }^{16}$ Ao passo que o humor deprimido é causado pela diminuição dos níveis de serotonina. ${ }^{6}$ É importante avaliar a severidade dos sintomas emocionais e psicológicos apresentados, haja vista a repercussão dos mesmos na vida das pessoas. Um estudo realizado em 2015 com 232 mulheres avaliou o impacto dos sintomas emocionais e mostrou que 50\% dos indivíduos com humor deprimido ou alterações emocionais, tentou suicídio. ${ }^{15}$

Com relação às consequências da SPM no cotidiano das acadêmicas, os estudos evidenciaram que os sintomas pré-menstruais trazem impactos negativos para o cotidiano. As consequências e impactos podem ser visualizados no âmbito do trabalho ou da faculdade, e nos relacionamentos familiares, fazendo com que as mulheres apresentem comportamentos hostis as pessoas, tenham a sensação de perder controle de suas ações, apresentem embotamento afetivo e isolamento. ${ }^{10,2}$

Um estudo que avaliou as consequências da forma mais grave de SPM, a TDPM em universitárias mostrou que os sintomas são responsáveis por uma série de consequências como: absenteísmo no trabalho/universidade, déficit na memória de trabalho, diminuição das funções cognitivas. $^{18}$

\section{Alternativas para controle dos sintomas}

Muitos estudos também avaliaram a efetividade de técnicas ou de alternativas para melhoria dos sintomas da SPM, sendo que as alternativas identificadas variavam desde o uso da fitoterapia até o processo de compra e consumismo. Um estudo de caso e controle realizado no Egito com estudantes de enfermagem mostrou que a técnica aromaterapia (que consiste na massagem abdominal com óleos essenciais), era eficaz no controle dos sintomas, principalmente na cólica, edema e sangramento excessivo. ${ }^{16}$

Outro estudo, que avaliou a eficácia do Mat Pilates, demonstrou que após as sessões de pilates, as mulheres obtiveram escores melhores quando comparados às que não fizeram as 
sessões de pilates. A melhora foi observada nos seguintes sintomas: irritabilidade, má concentração, cólicas, insônia, ansiedade, depressão, edema e aumento na vontade do apetite. ${ }^{11}$

Um estudo brasileiro, relacionado ao mercado consumidor mostrou que o desejo de compras no período pré-menstrual, pode ser considerado como uma alternativa para controle de alguns sintomas emocionais, como por exemplo, a melhora do auto-estima e humor deprimido, como pode ser observado ao analisar o trecho a seguir:

\footnotetext{
"Estas mulheres experimentaram uma necessidade de consumo, de adquirir algo, de suprir uma falta. Algumas das entrevistadas expressaram isto claramente. Nesta condição, as consumidoras simplesmente vão à compra. Para algumas, a vontade de comprar, a necessidade de se sentirem melhor consigo mesmas, se se sentirem mais bonitas, ou menos feias. No mundo imaginário destas mulheres, algo do mundo exterior que elas podem comprar, roupas, sapatos ou acessórios, promete o resgate da beleza ou o preenchimento daquele vazio. ${ }^{17: 71}$
}

Também foi mostrada a efetividade da Terapia Cognitiva Comportamental (TCC), em estudo realizado na Alemanha em 2014. As mulheres que sofriam com sintomas da SPM participaram do programa de intervenção que durou 8 semanas, o tratamento consistia seguir as orientações dos 14 módulos oferecidos pelo programa, que eram acessados de casa por meio da internet. O primeiro módulo incluía psicoeducação sobre causas e tratamento para SPM. Sugestões, como prática de atividades físicas, alimentação saudável estavam incluídas. Já o último módulo consistia em uma revisão de todos os outros e prevenção de recaída. ${ }^{1}$

Para ter controle de adesão das pacientes ao tratamento, e-mails semanais eram necessários. De acordo o estudo os benefícios deste programa para as mulheres, consistia na seriedade e validade com que os sintomas que obtiveram melhora eram avaliados, além da mudança do estilo de vida percebido. ${ }^{1}$

\section{Considerações Finais}

Após análise dos artigos encontrados, pode-se concluir que a SPM é uma síndrome caracterizada por sintomas físicos, em que as cólicas e edema são mais prevalentes e sintomas emocionais, em que a irritabilidade e humor deprimido ganham destaque. 
Id on Line Revista Multidisciplinar e de Psicologia

Id on Line Multidisciplinary and Psycology Journal

Todos os sintomas apresentados pelas mulheres acarretam graves prejuízos no contexto social (família/trabalho/estudo) e contexto pessoal. O que indica que a SPM é uma síndrome que requer avaliação criteriosa. Os estudos também demonstraram a variedade e eficácia de algumas terapias para controle dos sintomas da SPM.

Dentre as limitações do estudo, cabe destacar o pequeno número de publicações específicas sobre as consequências dos sintomas da SPM em universitárias. Inúmeros estudos avaliam as consequências da SPM na população de mulheres em geral, no entanto, poucos estudos são direcionados para a população universitária, deixando um vasto espaço neste aspecto. Deste modo propõe-se a elaboração de um número maior de pesquisas direcionadas para este público.

\section{Referências}

1 Silveira A da, Vieira E, Leão DM, Nicorena BPB, Rodrigues FR, Sandoval Longoria, E. Síndrome da tensão pré-menstrual observada em usuárias do ambulatório municipal de saúde da mulher. Enfermeria Global. 2014; 35:74-84

2 Dutra C. Repercussões da síndrome pré-menstrual na vida da mulher. Monografia (Enfermagem). Porto Alegre/RS, 2015.

3 Berek J. et al. Tratado de Ginecologia. 14 ${ }^{\text {a }}$ Ed. Rio de Janeiro: Guanabara Koogan, 2012.

4 Santos L. et al. Estado nutricional e consumo alimentar de mulheres jovens na fase lútea e folicular do ciclo menstrual. Rev. nutr. 2011; 24(2): 323-331.

5 Maia M, de Aguiar MIF, Chaves ES, Rolim ILTP. Qualidade de vida de mulheres com tensão pré-menstrual a partir da escala WHOQOL-BREF. Cienc Cuid Saude. 2014; 13(2):236-244.

6 Diniz MS da, Lima ACS, Pereira H, Ferreira G. Prevalência da Síndrome Pré-menstrual e seus principais sintomas observados em acadêmicas do Curso de Medicina de uma faculdade do sul de Minas Gerais. Revista Ciências em Saúde. 2013; 3(2):17p.

7 Gil AC. Como elaborar projetos de pesquisa. 4. ed. São Paulo: Atlas, 2008.

8 Bardin L. L'analyse de contenu, 1 ${ }^{\text {a }}$ edição. Lisboa. Presses Universitária France; 1977.

9 Kues JN, Janda C, Kleinstauber M, Weise C. Internet-based cognitive behavioural self-help for pré-menstrual syndrome: study protocol for a randomised controlled trial. Trials Journal Online. 2014; 15(1):9p 
Id on Line Revista Multidisciplinar e de Psicologia

Id on Line Multidisciplinary and Psycology Journal

10 Luz JM, Osório SMB, Santos IMS de, Piauilino YML, de Barros VL, Nicola AIO. Consequências da Síndrome Pré-menstrual em acadêmicas de enfermagem. RETEP - Rev. Tendên. da Enferm. Profis. 2015; 7(2): 1537-1541

11 Oliveira TJ. Oliveira C. Influência do método MAT Pilates no tratamento da síndrome da tensão pré-menstrual. $15^{\circ}$ Congresso Nacional de Iniciação Científica. São Paulo, 2011.

12 Sousa LWS, Damasceno AKC, Lopes EM, Aquino PS de. Fatores relacionados à síndrome pré-menstrual em acadêmicas de enfermagem. remE - Rev. Min. Enferm. 2010;14(4): 548-553.

13 Câmara RA, Síndrome Pré-Menstrual Estudo de prevalência em alunas da Universidade da Beira Interior. Dissertação apresentada a Faculdade de Ciências da Saúde - Universidade da Beira Interior. Corvilhã - Portugal. 2011

14 Tolossa FW, Bekele ML. Prevalence, impact and medical managenements of premenstrual syndrome among female students: cross-sectional study in college of health sciences, Mekelle University, Mekelle, Northen Ethiopia. Women's Health 2014, 14:52 http://www.biomedcentral.com/1472-6874/14/52

15 Ducasse D, Jaussent I, Olié E, Guillaume S, Lopez-Castroman J, Courtet P Personality Traits of Suicidality Are Associated with Premenstrual Syndrome and Premenstrual Dysphoric Disorder in a Suicidal Women Sample. PLoS ONE. 2016; 11(2): e0148653. doi:10.1371/journal.pone.0148653

16 Marzouk TMF, El-Nemer AMR, Baraka HN. The Effect of Aromatherapy Abdominal Massage on Alleviating Menstrual Pain in Nursing Students: A Prospective Randomized CrossOver Study. 2013, Article ID 742421, 6 pages.

17 Lara JE, Barbosa AAR, Pinto MR. Mulheres, tensão pré-menstrual e compras: Freud explica? Pretexto. 2015; 16(3): 58-76.

18 Almeida CGF. Associação entre Síndrome Disfórica pré-menstrual, atenção, memória e aprendizagem de estudantes de medicina. Universidade Federal do Ceará. Dissertação de Mestrado, Fortaleza; 2014.

\section{Como citar este artigo (Formato ABNT):}

MENDES, N.A.; SPUZA, A.P. Alterações Fisiológicas Relacionadas À Síndrome Da Tensão Pré-Menstrual Na Vida Da Mulher. Id on Line Revista Multidisciplinar e de Psicologia, Janeiro de 2017, vol.10, n.33, p. 01-09. ISSN: 1981-1179.

Recebido: 23/11/2016

Aceito: $24 / 11 / 2016$ 